\title{
Friction on a Quantized Vortex in a Superfluid
}

\author{
X.-M. Zhu ${ }^{a}$ and P. Ao ${ }^{b \dagger}$ \\ Department of Experimental ${ }^{a} /$ Theoretical $^{b}$ Physics \\ Umeå University, S-901 87, Umeå, SWEDEN
}

November 4, 2018

\begin{abstract}
We obtain the explicit expression of the friction on a moving quantized vortex, following the formulation of Thouless, Ao and Niu. It is shown that the friction on a moving vortex is sensitive to details but does not change the transverse force. We provide a general thermodynamic interpretation for the mutual independence of the transverse force and the friction. The friction is evaluated for the case of quasiparticle contributions in a clean fermionic superfluid, showing a new feature of logarithmic divergence.
\end{abstract}

PACS\#s: $67.40 . V s ; 47.37 .+8$

In physical systems few qualities have generic values independent of material details. When such cases occur, usually there are either symmetry or topology reasons [1]. The controversy over dynamics of quantized vortices in superfluids or superconductors 2, 203 may be partly understood as due to the apparent conflict between the exact results [4, 5, 6] with the existence of various scattering processes in the system. When a vortex moves, in addition to the hydrodynamic Magnus force which is proportional to the superfluid density, the coupling between the vortex to quasiparticles, phonons and impurities may cause extra forces in both transverse and longitudinal directions of the vortex motion. Those possible non-vanishing forces have always been the underlying assumption for phenomenological models used in analyzing experiments[2], 迆. 
While the transverse force on a moving vortex is controversial, the friction is poorly studied. The usual quoted friction formula derived from a microscopic Hamiltonian is obtained through a relaxation time approximation in force-force correlation functions. However, this type of procedure has been known to be wrong since 60's, in the context of obtaining the friction of electrons, i.e. resistivity. [8] The lack of explicit and correct microscopic derivation of friction has two consequnces. First, though the friction of vortex motion is an important quantity in many experiments, it has not been received its due attention. Second, the incomplete understanding of the friction has generated doubts on the exact result on the transverse force.

A self-contained theory of both the friction and the transverse force by a rigorous and elementary method is undoubtedly desirable. To provide such a calculation in an explicit and complete manner is the main purpose of the present letter. The method we choose follows that of Ref. [5]. In order to obtain the friction, we use a limiting process similar to the one in the transport theory and in non-equilibiurm statistical mechanics. This limiting process has only been mentioned but not explicitly discussed in Ref. [5] due to its sensitivity to details.

In the following, after giving a general expression for the friction, as an example, we evaluate the friction for the case of a clean fermionic superfluid. The resulting friction is completely new. It comes from the off-diagonal potential scattering of the extended quasiparticles, and is stronger than the ohmic damping by a logarithmic diverging factor. This new result illustrates the directness and usefulness of our formulation, another purpose of the present paper.

We consider an isolated vortex in the superfluid whose position $\mathbf{r}_{0}$ is specified by a pinning potential. The system is otherwise homogeneous and infinite. There is no externally applied supercurrent or normal current. The vortex is allowed to move slowly. Hence the system Hamiltonian $H$ contains a slow varying parameter $\mathbf{r}_{0}(t)$. The many body wavefunction of the superfluid $\left|\Psi_{\alpha}(t)\right\rangle$ can be expanded in terms of the instantaneous eigenvalues $E_{\alpha}\left(\mathbf{r}_{0}\right)$ and eigenstates $\left|\psi_{\alpha}\left(\mathbf{r}_{0}\right)\right\rangle$, for which we choose phases such that $\left\langle\psi_{\alpha} \mid \dot{\psi}_{\alpha}\right\rangle=0$. Because of our assumption of a homogeneous superfluid, $E_{\alpha}\left(\mathbf{r}_{0}\right)$ is independent of both $\mathbf{r}_{0}$ and time $t$. With those considerations, the many- 
body wavefunction $\left|\Psi_{\alpha}(t)\right\rangle$ can be expressed by

$$
\left|\Psi_{\alpha}(t)\right\rangle=e^{-i E_{\alpha} t / \hbar}\left|\psi_{\alpha}\left(\mathbf{r}_{0}\right)\right\rangle+\sum_{\alpha^{\prime} \neq \alpha} a_{\alpha^{\prime}}(t) e^{-i E_{\alpha^{\prime}} t / \hbar}\left|\psi_{\alpha^{\prime}}\left(\mathbf{r}_{0}\right)\right\rangle
$$

where, to the first order in velocity, $a_{\alpha}(t)=1$, and

$$
a_{\alpha^{\prime}}(t)=-\int_{0}^{t} d t^{\prime}\left\langle\psi_{\alpha^{\prime}} \mid \dot{\psi}_{\alpha}\right\rangle e^{i\left(E_{\alpha^{\prime}}-E_{\alpha}\right) t^{\prime} / \hbar}
$$

Here $\nabla_{0}$ denotes the partial derivative with respect to the position $\mathbf{r}_{0}$ of the pinning potential, the vortex position. This gives the expectation value of the force on the vortex as

$$
\begin{aligned}
\mathbf{F}= & -\sum_{\alpha} f_{\alpha}\left\langle\Psi_{\alpha}\left|\nabla_{0} H\right| \Psi_{\alpha}\right\rangle \\
= & -\sum_{\alpha} f_{\alpha}\left\langle\psi_{\alpha}\left|\nabla_{0} H\right| \psi_{\alpha}\right\rangle \\
& +\sum_{\alpha^{\prime} \neq \alpha} f_{\alpha}\left\langle\psi_{\alpha}\left(\mathbf{r}_{0}(t)\right)\left|\nabla_{0} H\right| \psi_{\alpha^{\prime}}\left(\mathbf{r}_{0}(t)\right)\right\rangle \times \\
& \int_{0}^{t} d t^{\prime}\left\langle\psi_{\alpha^{\prime}}\left(\mathbf{r}_{0}\left(t^{\prime}\right)\right) \mid \dot{\psi}_{\alpha}\left(\mathbf{r}_{0}\left(t^{\prime}\right)\right)\right\rangle e^{i\left(E_{\alpha^{\prime}}-E_{\alpha}\right)\left(t^{\prime}-t\right) / \hbar}+c . c .,
\end{aligned}
$$

where $f_{\alpha}$ is the occupation probability of the state $\alpha$. For a vortex moving with a small and uniform velocity $\mathbf{v}_{V}$,

$$
\left|\dot{\psi}_{\alpha}\left(\mathbf{r}_{0}\right)\right\rangle=\mathbf{v}_{V} \cdot\left|\nabla_{0} \psi_{\alpha}\left(\mathbf{r}_{0}\right)\right\rangle
$$

The first term in the right hand side of Eq.(3) is independent of $\mathbf{v}_{V}$ and will be ignored. In fact, it is zero because of the translational invariance regarding to the vortex position. The integration in time can be carried out directly. In addition, we also need to use the following relations,

$$
\left\langle\psi_{\alpha}\left|\nabla_{0} H\right| \psi_{\alpha^{\prime}}\right\rangle=\left(E_{\alpha^{\prime}}-E_{\alpha}\right)\left\langle\psi_{\alpha} \mid \nabla_{0} \psi_{\alpha^{\prime}}\right\rangle=\left(E_{\alpha}-E_{\alpha^{\prime}}\right)\left\langle\nabla_{0} \psi_{\alpha} \mid \psi_{\alpha^{\prime}}\right\rangle
$$

which are obtained by taking gradient $\nabla_{0}$ with respect to $H\left|\psi_{\alpha^{\prime}}\right\rangle=E_{\alpha^{\prime}}\left|\psi_{\alpha^{\prime}}\right\rangle$ and $\left\langle\psi_{\alpha}\right| H=E_{\alpha}\left\langle\psi_{\alpha}\right|$, then multiplying from left/right by $\left\langle\psi_{\alpha}\right|$ or $\left|\psi_{\alpha^{\prime}}\right\rangle$ respectively. After making simplifications by using Eq.(5), Eq.(3) gives

$$
\mathbf{F}=\frac{1}{2} \sum_{\alpha^{\prime} \neq \alpha} \frac{f_{\alpha}-f_{\alpha^{\prime}}}{E_{\alpha}-E_{\alpha^{\prime}}} \frac{\sin \left(E_{\alpha}-E_{\alpha^{\prime}}\right) t / \hbar}{\left(E_{\alpha}-E_{\alpha^{\prime}}\right) / \hbar}\left|\left\langle\psi_{\alpha}\left|\nabla_{0} H\right| \psi_{\alpha^{\prime}}\right\rangle\right|^{2} \mathbf{v}_{V}
$$


$+i \hbar \sum_{\alpha^{\prime} \neq \alpha} f_{\alpha}\left(1-\cos \left(E_{\alpha}-E_{\alpha^{\prime}}\right) t / \hbar\right)\left\{\left(\left\langle\psi_{\alpha} \mid \nabla_{0} \psi_{\alpha^{\prime}}\right\rangle \times\left\langle\nabla_{0} \psi_{\alpha^{\prime}} \mid \psi_{\alpha}\right\rangle\right) \cdot \hat{\mathbf{z}}\right\} \mathbf{v}_{V} \times \hat{\mathbf{z}}$

the longitudinal and transverse forces on a moving vortex.

To obtain the long time behavior of Eq.(6) requires a limiting procedure. There are two ways of taking limiting sequences. A possible sequence is to take the low frequency limit before the thermodynamic limit. As we will find out, the transverse force is independent of the limiting process. Therefore such a limiting sequence is correct as long as only the transverse force is concerned. In such a calculation, because the energy levels have been treated as discrete ones, there is no friction. In order to obtain friction, we have to take the thermodynamic limit before the low frequency limit, the correct limiting procedure. For this purpose, we use the Laplace average 9], $\lim _{t \rightarrow \infty} \mathbf{F}(t)=\lim _{\epsilon \rightarrow 0^{+}} \mathbf{F}(\epsilon)$, where $\mathbf{F}(\epsilon)=\epsilon \int_{0}^{\infty} d t \mathbf{F}(t) e^{-\epsilon t}$. We will also use the identity $1 /(\epsilon+i x)=\pi \delta(x)-i P(1 / x)$. For the transverse force $\mathbf{F}_{\perp}$, we have

$$
\lim _{\epsilon \rightarrow 0^{+}} \epsilon \int_{0}^{\infty} d t e^{-\epsilon t}\left(1-\cos \left(E_{\alpha}-E_{\alpha^{\prime}}\right) t / \hbar\right)=\lim _{\epsilon \rightarrow 0^{+}}\left(1-\pi \delta\left(E_{\alpha}-E_{\alpha^{\prime}}\right) \epsilon\right)=1,
$$

because the summation over states in Eq.(6) is well behaved regardless whether $E_{\alpha}$ is discrete or continuous. Hence

$$
\mathbf{F}_{\perp}=i \hbar \sum_{\alpha^{\prime} \neq \alpha} f_{\alpha}\left\{\left(\left\langle\psi_{\alpha} \mid \nabla_{0} \psi_{\alpha^{\prime}}\right\rangle \times\left\langle\nabla_{0} \psi_{\alpha^{\prime}} \mid \psi_{\alpha}\right\rangle\right) \cdot \hat{\mathbf{z}}\right\} \mathbf{v}_{V} \times \hat{\mathbf{z}}
$$

This is precisely what has been obtained in Ref. [5], where further calculations lead to $\mathbf{F}_{\perp}=-h L \rho_{s} \mathbf{v}_{V} \times \hat{\mathbf{z}}$, independent of details of the system. Here $\rho_{s}$ is the superfluid number density, $L$ the length of the vortex. The longitudinal force, friction, is given by $\mathbf{F}_{\|}=-\eta \mathbf{v}_{V}$ with

$$
\eta=\frac{\pi}{2} \sum_{\alpha^{\prime} \neq \alpha} \hbar \frac{f_{\alpha}-f_{\alpha^{\prime}}}{E_{\alpha^{\prime}}-E_{\alpha}} \delta\left(E_{\alpha}-E_{\alpha^{\prime}}\right)\left|\left\langle\psi_{\alpha}\left|\nabla_{0} H\right| \psi_{\alpha^{\prime}}\right\rangle\right|^{2} .
$$

The coefficient of friction $\eta$ is determined by low energy excitations such as phonons, extended quasiparticles, and localized quasiparticles when their discrete energy spectrum is smeared out by impurities. This expression is identical to the result in Ref. [6] for the case of ohmic damping in the zero frequency limit. Eq.(8) will not pick up any superohmic contributions, and 
will give infinity for any subohmic contributions. We point out that while with the aid of Eq.(5) the transverse force can be expressed only in terms of the wavefunction or the density matrix without explicitly refering to the Hamiltonian or its eigenvalues, as showing by Eq.(7), the longitudinal force, the friction, cannot, as showing by Eq.(8). The explicit dependence on the Hamiltonian or its eigenvalues in Eq.(8) is the source of the sensitivity of friction to details of the system.

For the superfluid ${ }^{4} \mathrm{He}$, there is no full microscopic theory yet [7]. We will not attempt to evaluation of Eq.(8) for this superfluid here because of the sensitivity of the friction to details. The situation can be very different in the case of the recent Bose-Einstein condensed systems, where a well defined microscopic theory is supposed to be known. Instead, as an example to illustrate the directness and usefulness, we evaluate Eq.(8) for the case of a homogeneous fermionic superfluid using BCS theory with s-wave pairing. At finite temperatures the extended states above Fermi level, the quasiparticles, are partially occupied. The vortex motion causes transitions between these states and gives rise to friction. The transitions between different single quasiparticle levels $\left\langle\psi_{\alpha}\left|\nabla_{0} H\right| \psi_{\alpha^{\prime}}\right\rangle$ are considered here since they dominate the low energy process. The quasiparticles are described by the eigenstates, $u_{\alpha}$ and $v_{\alpha}$, of the Bogoliubov-de Gennes equation. Their behaviors in the presence of a vortex has been well studied in Ref. 10. We may take

$$
\left|\psi_{\alpha}\right\rangle=\left(\begin{array}{c}
u_{\alpha}(x) \\
v_{\alpha}(x)
\end{array}\right)=\frac{1}{\sqrt{L}} e^{i k_{z} z} e^{i \mu \theta+i \sigma_{z} \theta / 2} \hat{f}(r),
$$

with $\mathbf{r}$ measured from the vortex position, and $\theta$ the azimuthal angle around the vortex. In order to obtain an analytical form for the transition element, we use a WKB solution for $\hat{f}(r)$,

$$
\begin{aligned}
\hat{f}(r)= & \frac{1}{2 \sqrt{R r}}\left(\begin{array}{l}
{\left[1 \pm \sqrt{E^{2}-|\Delta(r)|^{2}} / E\right]^{1 / 2}} \\
{\left[1 \mp \sqrt{E^{2}-|\Delta(r)|^{2}} / E\right]^{1 / 2}}
\end{array}\right) \times \\
& \exp \left\{i \int_{r_{t}}^{r} d r^{\prime}\left(k_{\rho}^{2} \frac{r^{\prime 2}-r_{t}^{2}}{r^{\prime 2}} \pm \frac{2 m}{\hbar^{2}} \sqrt{E^{2}-\left|\Delta\left(r^{\prime}\right)\right|^{2}}\right)^{1 / 2}\right\}+\text { c.c. }
\end{aligned}
$$

Here $k_{\rho}^{2}=k_{f}^{2}-k_{z}^{2}, R$ is the radial size of the system. This WKB solution is valid when $r$ is outside the classical turning point $r_{t}=|\mu| / k_{\rho}$. Here $r_{t}$ is the impact parameter. A WKB solution also exists inside the turning point. 
However, because it approaches zero as $\left(r k_{\rho}\right)^{|\mu|} /|\mu|$ !, the contribution to the transition elements from this region is small, and will be set to zero. The transition elements are then given by

$$
\begin{aligned}
\left|\left\langle\psi_{\alpha}\left|\nabla_{0} H\right| \psi_{\alpha^{\prime}}\right\rangle\right|^{2} & =\left|\int d x\left(u_{\alpha^{\prime}}^{*}(x) \nabla_{0} \Delta v_{\alpha}(x)+v_{\alpha^{\prime}}^{*}(x) \nabla_{0} \Delta^{*} u_{\alpha}(x)\right)\right|^{2} \\
& = \begin{cases}\frac{\Delta_{\infty}^{4}}{E^{2} R^{2}} \delta_{k_{z 1}, k_{z 2}} \delta_{\mu_{1}, \mu_{2} \pm 1}, & |\mu| \leq \xi_{0} k_{\rho} \\
0, & |\mu|>\xi_{0} k_{\rho}\end{cases}
\end{aligned}
$$

Here $\Delta_{\infty}$ is the value of $|\Delta(r)|$ far away from the vortex core. Physically it means that if the classical quasiparticle trajectory is far away from the vortex core, it will not contribute to the friction. The summation over states in Eq.(8) is replaced by

$$
\sum_{\alpha^{\prime} \neq \alpha}=\sum_{\mu_{1}, \mu_{2}, k_{z 1}, k_{z 2}} \int d E_{1} d E_{2} \frac{E_{1}}{\sqrt{E_{1}^{2}-\Delta_{\infty}^{2}}} \frac{E_{2}}{\sqrt{E_{2}^{2}-\Delta_{\infty}^{2}}}\left(\frac{m}{\hbar^{2} k_{f}}\right)^{2} \frac{R^{2}}{\pi^{2}},
$$

after considering the density of states.

Substituting Eq.(11) into Eq.(8), using the quasiparticle distribution function $f_{\alpha}=1 /\left(e^{\beta E_{\alpha}}+1\right)$, the coefficient of friction is given by

$$
\eta=\frac{L m^{2} \xi_{0} \Delta_{\infty}^{4} \beta}{8 \pi^{2} \hbar^{3}} \int_{\Delta_{0}}^{\infty} d E \frac{E^{2}}{E^{2}-\Delta_{\infty}^{2}} \frac{1}{E^{2} \cosh ^{2}(\beta E / 2)} .
$$

The integral in Eq.(12) diverges logarithmically. It implies that the spectral function corresponding to the vortex-quasiparticle coupling is not strictly ohmic but has an extra frequency factor proportional to $\ln \left(\Delta_{\infty} / \hbar \omega\right)[11$, 6]. When $\hbar \omega$ is not very small comparing to $\Delta_{\infty}$, which may be realized when close to $\mathrm{T}_{c}$, we can ignore the logarithmic divergence in Eq.(12) by using the density of states for normal electrons to obtain a finite friction, i.e. replacing $E^{2} /\left(E^{2}-\Delta_{\infty}^{2}\right)$ with 1 in Eq.(12). Close to $\mathrm{T}_{c}$, the friction approaches to zero the same way as $\Delta_{\infty}^{2}$, which is proportional to the superfluid density $\rho_{s}$. When $-\ln \left(\hbar \omega / \Delta_{\infty}\right)$ is large, we need to use a more accurate expression of vortex friction obtained in Ref. [6]. Straightforward evaluation shows that in such a case

$$
\eta=\frac{L m^{2} \xi_{0} \Delta_{\infty}^{3} \beta}{16 \pi^{2} \hbar^{3}} \frac{1}{\cosh ^{2}\left(\beta \Delta_{\infty} / 2\right)} \ln \left(\Delta_{\infty} / \hbar \omega_{c}\right) .
$$


Here $\omega_{c}$ is the low frequency cut-off. It is determined by the size of the system for a single vortex, and by the inter-vortex distance for a vortex array.

It should be emphasized that the logarithmic divergence comes from the interplaying between the divergence in the density of states and the offdiagonal potential scattering. We can consider a situation that we physically create a pinning center to trap the vortex and guide its motion. In such a case the vortex has a diagonal potential. If the scattering is dominated by the diagonal potential, e.g., by the pinning potential, additional factor coming from $\left|u_{\alpha}\right|^{2}----\left|v_{\alpha}\right|^{2}$ will remove this logarithmic divergent. This again shows the sensitivity of the friction to system details.

This extended state friction contribution exists for both clean and dirty superconductors at finite temperatures. Close to the transition temperature, it scales linearly with the superfluid density, and is exponentially small when $T<<\Delta_{\infty}$. For intermediate temperature $T \sim \Delta_{\infty}$, using $\xi_{0} \sim \hbar^{2} k_{F} / m \Delta_{\infty}$ and $N(0)=m k_{F} / \pi^{2} \hbar^{2}, \eta \sim L \hbar N(0) \Delta_{\infty}^{2} / k_{B} T$. For dirty superconductors additional contribution to the friction arise from vortex core states. This contribution, which remains finite at zero temperature, was first discussed phenomenologically in Ref. [12]. Eq.(12) also permits us to do a detailed microscopic calculation for the core contributions, as pointed out in Ref.[6]. Another application of the above results may be the dynamics of the axisymmetric vortex in ${ }^{3} \mathrm{He} \mathrm{B}$ phase, where the Bogoliubov-de Gennes equation is essentially the same as the one in a s-wave superconductor 13.

As we have shown, the friction on moving vortex has certain interesting features which can be tested experimentally. It is also clear that transverse force is not influenced by the existence of a friction. This is significant different from another familiar example, the quantum Hall system, where it is generally understood that the energy gap in the quasiparticle excitation spectrum stabilizes the topological structure, meanwhile makes the dissipation to vanish. When the quantized Hall conductance occurs it associates with the vanishing of longitudinal voltage. However, the total transverse force on a moving quantized vortex, which has a topological significance, remains unchanged with finite friction.

There has been a great amount of effort in this subject, because this is the only type of topological singularities which can be extensively studied experimentally, in both classical and quantum regimes. Some of the previous work on vortex motion in fermionic superfluid has been discussed critically in [6, 8]. In bosonic superfluids, the situation of early work is more diverse[7]. 
So far, the experimental papers on superfluids and superconductors claim support to different theoretical results 14, 15, 16, 17. We need to keep in mind that in general the real experimental situations are far more complicated than ones usually considered in theoretical models. For example, in the analysis of Hall measurements in superconductors, the vortex interaction and the pinnings are often ignored. If we use the same assumption for longitudinal resistivity, we would conclude that we should have flux flow for any current and at any temperature, which contradicts the longitudinal resistivity measurements [2]. Complications also exist in mutual friction measurements. For example, the additional friction caused by the relative motion between the ends of the vortex lines and the diaphragm has not been considered in a recent experiment [15]. In addtion, because these vortex lines are shorter than the viscous penetration depth of the normal fluid, the boundary effect can also be important. Conclusions on the transverse force drawed from such experimental data should be taken with a due caution.

Before we conclude, we discuss the thermodynamic interpretation of our results. We have shown that indeed a finite friction does not change the transverse force. To illustrate this, let us imagine a torus-shaped tank filled with superfluid. The normal fluid is assumed to be at rest with the tank initially. After creating a vortex-antivortex pair, we apply an external force on the vortex to let it wind around one of the circumference of the torus once with uniform velocity in time $t_{\text {total }} \rightarrow \infty$ before their annihilation. We consider the work done by the external force to the system, which includes the superfluid, normal fluid and the tank(subtrate), and the energy and entropy gain of the system. The vortex is used as an intermediate step to find the forces. The external force on the vortex in the longitudinal direction balances the force felt by the vortex due to its coupling to the normal fluid. The external force on the vortex in the transverse direction balances the total force on the superfluid, which is the summation of the Mangus force and a possible one from the normal fluid.

The superfluid velocity has a finite increase due to the phase change caused by the vortex motion. Thus the kinetic energy of the superfluid also increases by a finite amount. This kinetic energy increase is exactly equal to the work done by the external force on the superfluid, if it is the Magnus force. The frictions do not dissipate energy, because the process is quasi-static in which the normal fluid velocity is negligible. Under such a condition, the external force on the superfluid in the transverse direction needs to be at 
least the magnitude of the Magnus force in order to provide the work for the increase of kinetic energy of the superfluid. The normal fluid and substrate cannot tranfer energy to the superfluid, which carries no entropy, by lowering their internal energies, because of the second law of thermodynamics 18 .

Our argument has not told us why the total transverse force is not larger than the Magnus force. If there is no entropy change in the normal fluid, then the transverse force will not be larger than the Magnus force because the energy increase in the system, carried by the superfluid, not normal fluid, must equal to the work done by the external forces. This is possible, because the superfluid carries zero entropy, and because our process is quasi-static, in which there is no entropy generation within the system. There is also no external heat bath to provide a heat flow. The entropy stays constant within the system.

In summary, both the transverse force and friction on a moving quantized vortex has been obtained simultaneously within the framework of Ref. [5]. The transverse force confirms the Berry phase results obtained with a trial many-body wavefunction[4]. New results on the friction are given for a fermionic superfluid, which can be tested experimentally.

Acknowledgments We thank David Thouless for useful comments. This work was financially supported by Swedish NFR.

†Present address: Bartol Research Institute, University of Delaware, Newark, DE 19716, USA

\section{References}

[1] D.J. Thouless, Topological Quantum Numbers in Nonrelativistic Physics, World Scientific, Singapore, 1998.

[2] E.H. Brandt, Rep. Prog. Phys. B58, 1465 (1995).

[3] For a recent critical review, see D.J. Thouless, P. Ao, and Q. Niu, M.R.Geller, and C. Wexler, Quantized Vortices in Superfluids and Superconductors, e-print: cond-mat/9709127.

[4] P. Ao and D.J. Thouless, Phys. Rev. Lett. 70, 2158 (1993).

[5] D.J. Thouless, P. Ao, and Q. Niu, Phys. Rev. Lett. 76, 3758 (1996). 
[6] P. Ao and X.-M. Zhu, Physica C282-287, 367 (1997). (e-print: condmat/9704247).

[7] R.J. Donnelly, Quantized Vortices in Helium II, Cambridge University Press, Cambridge, 1991.

[8] X.-M. Zhu and P. Ao, Chinese J. Phys. (in press) (e-print: condmat/9709311).

[9] W. Kohn and J.M. Luttinger, Phys. Rev. 108, 590 (1957).

[10] J. Bardeen, R. Kümmel, A.E. Jacobs, and L. Tewordt, Phys. Rev. 187, 556 (1969).

[11] A.O. Caldeira and A.J. Leggett, Ann. Phys.(NY) 149, 374 (1983).

[12] J. Bardeen and M.J. Stephen, Phys. Rev. 140, A1197 (1965).

[13] D. Vollhardt and P. Wölfle, The Superfluid Phases of Helium 3, Taylor \& Francis, London, 1990.

[14] X.-M. Zhu, E. Brandstrom, and B. Sundqvist, Phys. Rev. Lett. 78, 122 (1997).

[15] J.R. Hook, T.D.C. Bevan, A.J. Manninen, J.B. Cook, A.J. Armstrong, and H.E. Hall, Physica B 210, 251 (1995).

[16] W.F. Vinen, Proc. Roy. Soc. (London), A 260, 218 (1961).

[17] R.J. Zieve, Yu. M. Mukharsky, J.D. Close, J.C. Davis, and R.E. Packard, J. Low Temp. Phys. 91, 315 (1993).

[18] H.B. Callen, Thermodynamics, Wiley, New York, 1960. 